\title{
Chlamydia felis exposure in companion dogs and cats in Lanzhou, China: a public health concern
}

\author{
Song-Ming $\mathrm{Wu}^{1+}$, Si-Yang Huang ${ }^{1 *+}$, Min-Jun Xu', Dong-Hui Zhou' ${ }^{1}$, Hui-Qun Song ${ }^{1}$ and Xing-Quan Zhu ${ }^{1,2}$
}

\begin{abstract}
Background: Chlamydiaceae is a family of obligate intracellular pathogens with a worldwide distribution in many animal species, including humans. No information exists on the prevalence of Chlamydia felis infections in cats and dogs in Lanzhou, the geographical center of China. The aim of this study was to carry out a census of cats and dogs in Lanzhou and document the seroprevalence of $C$. felis exposure in these companion animals.

Results: In this study, blood samples were collected from 485 animals (221 cats and 264 pet dogs) in Lanzhou between November 2010 and July 2011 to identify antibodies against C. felis. Thirteen of 221 (5.9\%) cats and 32 of $264(12.1 \%)$ pet dogs were positive for $C$. felis infection using indirect hemagglutination at a cutoff of 1:16. The seroprevalence in household and stray cats was $3.9 \%$ and $14.3 \%$, respectively, and the difference was statistically significant $(P<0.05)$. Among different age groups, the seroprevalence in cats varied from 1.9 to $7.9 \%$, and that in dogs ranged from 9.6 to 20.4\%; however, the differences were not statistically significant $(P>0.05)$.

Conclusions: This is the first report of the seroprevalence of $C$. felis exposure in cats and dogs in Lanzhou, northwestern China. Our results indicate that the presence of $C$. felis exposure in cats and dogs may pose a potential threat to human health.
\end{abstract}

Keywords: Chlamydia felis, Chlamydiosis, Survey, Indirect hemagglutination (IHA) test, Cats, Dogs

\section{Background}

Chlamydia is a genus comprising important zoonotic obligate intracellular pathogens that affect humans and a wide range of animals, including birds [1,2]. Chlamydia infection causes a wide spectrum of diseases in nonhuman mammals and birds, including atypical pneumonia, enteritis, conjunctivitis, endocarditis, and even abortion, resulting in heavy economic losses [3-6]. Several Chlamydia species are transmissible to humans and are of serious public health significance because they may lead to pneumonia, atherosclerosis, coronary heart disease, and other severe diseases. Chlamydia abortus and C. psittaci are of particular importance because they can cause abortion and psittacosis, respectively, in animals, birds and humans. The animal diseases caused by these microorganisms should be given more attention in terms of their

\footnotetext{
* Correspondence: siyang.huang@hotmail.com

${ }^{\dagger}$ Equal contributors

'State Key Laboratory of Veterinary Etiological Biology, Lanzhou Veterinary Research Institute, Chinese Academy of Agricultural Sciences, Lanzhou, Gansu Province 730046, PR China

Full list of author information is available at the end of the article
}

zoonotic aspects and the zoonotic potential of other animal pathogens [2,7-9].

Chlamydia felis is an important agent with zoonotic potential. It causes primary infections in the upper respiratory tract and eyes of cats. It is usually transmitted through the air and in secretions from infected cats' eyes or noses [10]. Clinical signs in dogs are similar to those associated with canine distemper, such as conjunctivitis, encephalitis, pneumonia, and keratitis [11,12]. Pet cats and dogs are considered to be faithful friends and companions of humans, thus playing an important role in human life. Unfortunately, cats and dogs may be important sources of C. felis infection in humans [8].

A study in China reported that severe pneumonia in peacocks and peacock farmers was caused by $C$. psittaci [13]. Another study indicated that 95 of 455 (20.9\%) Tibetan sheep were seropositive for $C$. abortus antibodies at the cutoff of 1:16 [14], and the seroprevalence of Chlamydia infection in Tibetan pigs in Tibet was 16.63\% between April and December 2010 [15]. Zhou et al. reported that the overall seroprevalence of chlamydial infection in dairy cattle was $7.25 \%$ in Guangzhou 
[16]. Xu et al. reported that $30.78 \%$ of pigs were positive for Chlamydia in Guangdong Province between March 2008 and May 2009 [12]. Surveys of C. felis infection in cats have been extensively reported worldwide [10], but limited information on C. felis infection in dogs is available. The present study was undertaken to characterize C. felis exposure and measure its seroprevalence in cats and pet dogs in Lanzhou, northwest China.

\section{Methods}

The cats and pet dogs examined in the present study were handled in accordance with the Good Animal Practice requirements of the Animal Ethics Procedures and Guidelines of the People's Republic of China. This study was approved by the Animal Ethics Committee of Lanzhou Veterinary Research Institute, Chinese Academy of Agricultural Sciences (Approval No. LVRIAEC2010-010). Informed client consent was obtained from the owners of all cats and pet dogs, and the study adhered to the highest standard (best practice) of veterinary care.

\section{Cat and dog serum samples}

Blood samples were collected from randomly selected cats and dogs between November 2010 and July 2011 in Lanzhou, northwest China. These cats and pet dogs were admitted to pet hospitals located in four districts of Lanzhou (Chengguan, Anning, Xigu and Qilihe districts). Some required blood sample examination, and half of these blood samples were randomly selected for this study because we could not evaluate all of them. The examined pets were admitted to the pet hospitals for various diseases and health problems; the details of each animal's disease condition were not available. Pet owners were asked about the breed, age, gender and geographical origin, and the biometric data of stray cats were estimated based on body condition and dental age. Blood samples were slowly collected from either the jugular or cephalic vein into labeled plain red-top tubes. The samples were transported to the laboratory and kept at room temperature for $2 \mathrm{~h}$ to allow for clot formation. The blood-filled tubes were then centrifuged at $1500 \mathrm{~g}$ or $3000 \mathrm{rpm}$ for 5 to $10 \mathrm{~min}$. After centrifugation, it was ensured that the gel had formed a clear barrier between the cells and the serum. The serum samples were separated and stored at $-20^{\circ} \mathrm{C}$ until further analysis.

\section{Detection reagents}

Antibodies to C. felis in cats and dogs were measured using a commercially marketed indirect hemagglutination (IHA) kit purchased from the Lanzhou Veterinary Research Institute of the Chinese Academy of Agricultural Sciences. The sensitivity and specificity values for the testing kit used in this study have been validated by ministry of agriculture of the People's Republic of China (NY/T 562-2002).

\section{Detection procedure}

The detection procedure followed the manufacturer's instructions as previously described [12,14-17]. Briefly, sera were added to a 96-well V-bottomed polystyrene plate and diluted two-fold up to 1:2048, starting at 1:4. The detected antigen was added to each well, and the plate was then shaken slightly for 2 min followed by incubation at $37^{\circ} \mathrm{C}$ for $2 \mathrm{~h}$. IHA titers of $1: 16$ or higher with the formation of an agglutinated erythrocyte layer were considered to be positive [12,14-17]; sera with dubious results were retested. According to the manufacturer's instructions, 1:16 is the normal cutoff point for detecting a positive sample. If we had chosen 1:8, the false-positive rate would have been higher; if 1:32 had been chosen, the true-negative rate would have been lower. Positive (sera from infected dogs) and negative (sera from negative dogs) controls provided by the manufacturer were included in each test and assayed at the same dilutions of the sera samples.

\section{Statistical analysis}

We evaluated the univariate effects of gender, age, and types of cats on the presence of antibodies against $C$. felis with a Chi square test by the SPSS for Windows (Release 18.0 standard version, SPSS Inc., Chicago, Illinois). The differences were considered statistically significant when $P<0.05$. The exact binomial confidence interval for each group was calculated.

\section{Results}

In this study, serum samples were collected from a total of 221 cats (179 household cats and 42 strays) and 264 pet dogs in Lanzhou, northwest China, and the antibodies to $C$. felis were measured by the IHA test. Thirteen of $221(5.9 \%)$ examined cats were seropositive for C. felis infection, and 32 of 264 (12.1\%) pet dogs were found to be positive for C. felis infection at the cutoff 1:16 (Tables 1 and 2). Among different age groups, the seroprevalence in cats varied from 1.9 to $7.9 \%$, and that in dogs ranged from 9.6 to $20.4 \%$ (Tables 1 and 2). The seroprevalence in household and stray cats was 3.9 and $14.3 \%$, respectively; however, dogs were only grouped in one category (household pets). The samples were collected in four different animal hospitals in the same city. Because the city is not large, comparative analyses were not performed.

\section{Discussion}

Epidemiological data regarding the distribution of animal chlamydiosis in China are scarce, and most of them are on food animals such as sheep, cattle, and pigs 
Table 1 Seroprevalence of Chlamydia felis infection in household and stray cats by gender and age in Lanzhou, northwest China using indirect haemagglutination

\begin{tabular}{|c|c|c|c|c|c|c|c|c|c|c|c|c|}
\hline \multirow{3}{*}{$\begin{array}{l}\text { Cat } \\
\text { groups }\end{array}$} & \multicolumn{3}{|c|}{ Types of cat } & \multirow[t]{3}{*}{$95 \% \mathrm{Cl}$} & & & & \multirow[t]{3}{*}{$95 \% \mathrm{Cl}$} & \multicolumn{3}{|l|}{ Total } & \multirow[t]{3}{*}{$95 \% \mathrm{Cl}$} \\
\hline & \multicolumn{3}{|c|}{ Household cats } & & \multicolumn{3}{|l|}{ Stray cats } & & & & & \\
\hline & No. tested & No. positive & Prevalence (\%) & & No. tested & No. positive & Prevalence (\%) & & No. tested & No. positive & Prevalence (\%) & \\
\hline \multicolumn{13}{|l|}{ Gender } \\
\hline Male & 87 & 4 & 4.6 & $1.26-10.99$ & 17 & 1 & 5.9 & $0.15-28.89$ & 104 & 5 & 4.8 & $1.59-10.52$ \\
\hline Female & 92 & 3 & 3.3 & $0.68-8.96$ & 25 & 5 & 20 & $6.81-40.72$ & 117 & 8 & 6.8 & $3.04-12.37$ \\
\hline \multicolumn{13}{|l|}{ Age (years) } \\
\hline$<1$ & 41 & 0 & 0 & & 12 & 1 & 8.3 & $0.21-38.47$ & 53 & 1 & 1.9 & $0.04-10.00$ \\
\hline $1 \leq Y_{r}<2$ & 65 & 2 & 3.1 & $0.37-10.71$ & 24 & 5 & 20.8 & $7.12-41.96$ & 89 & 7 & 7.9 & $3.28-15.15$ \\
\hline $2 \leq Y_{r}<3$ & 37 & 2 & 5.4 & $0.66-18.39$ & 3 & 0 & 0 & & 40 & 2 & 5.0 & $0.61-17.19$ \\
\hline$\geq 3$ & 36 & 3 & 8.3 & $1.75-22.61$ & 3 & 0 & 0 & & 39 & 3 & 7.7 & $1.61-21.12$ \\
\hline Total & 179 & 7 & 3.9 & $1.60-7.84$ & 42 & 6 & 14.3 & $5.38-28.59$ & 221 & 13 & 5.9 & $3.20-9.56$ \\
\hline
\end{tabular}


Table 2 Prevalence of antibodies to Chlamydia felis in pet dogs by gender and age in Lanzhou, northwest China using indirect haemagglutination

\begin{tabular}{lllll}
\hline $\begin{array}{l}\text { Biometric } \\
\text { data }\end{array}$ & No. tested & No. positive & Prevalence (\%) & $\mathbf{9 5 \%} \mathbf{C l}$ \\
\hline Gender & & & & \\
Male & 139 & 14 & 10.1 & $5.72-15.85$ \\
Female & 125 & 18 & 14.4 & $9.22-20.81$ \\
Age (years) & & & & \\
$<1$ & 83 & 8 & 9.6 & $4.34-17.02$ \\
$1 \leq \mathrm{Yr}<2$ & 64 & 5 & 7.8 & $2.58-17.25$ \\
$2 \leq \mathrm{Yr}<3$ & 49 & 10 & 20.4 & $10.12-35.36$ \\
$\geq 3$ & 68 & 9 & 13.2 & $6.22-23.57$ \\
Total & 264 & 32 & 12.1 & $8.49-16.51$ \\
\hline
\end{tabular}

[14-16]. Companion animals, such as pet cats and dogs, are considered to be faithful friends of humans; however, cats and dogs could be important sources of Chlamydia infection in humans. Therefore, the present study aimed to estimate the chlamydial seroprevalence in cats and pet dogs in Lanzhou, northwest China. Several studies have reported the $C$. felis prevalence in cats in various regions. For example, Halánová $M$ et al. [18] used direct immunofluorescence and detected an overall $45.16 \%$ prevalence of C. felis among cats in Slovak, Millán, and Rodríguez [19] detected a $27 \%$ seroprevalence in serum samples from European wildcats using enzyme-linked immunosorbent assay. The overall seroprevalence of C. felis exposure in cats in Lanzhou was $5.9 \%$, which is lower than those in the above mentioned studies but higher than that observed in cats in Dongguan (2.38\%), southern China, using the same commercial IHA kit [20]. The differences in the seroprevalence of C. felis exposure in cats could be related to differences in ecological and geographical factors such as temperature, rainfall, or landscape differences; serologic tests used; feeding; and animal welfare protocols for cats.

Our results indicate that the seroprevalence of C. felis exposure in stray cats (14.3\%) was significantly higher than that observed in household cats $(3.9 \%)(P<0.05)$, which supports the results obtained by Halánová [18]. These differences are probably attributed to lifestyle and animal welfare protocols. Stray cats have more opportunities to come into contact with infected birds or other animals, and they suffer from poor nutrition and possibly compromised immune systems that may contribute to increased exposure to infectious pathogens. This may explain the higher $C$. felis prevalence in stray cats than in pet cats. One study reported that a large number of cats and dogs become unowned each year in the UK, which may have considerable implications for their welfare [21].
Studies using both culture and polymerase chain reaction (PCR) indicated that cats less than 1 year of age were the most likely to be infected with Chlamydia, and cats older than 5 years were the least likely to be infected [10]. However, although the differences were not statistically significant, we found a higher prevalence of antibodies to C. felis in older animals in this study. The $C$. felis seroprevalence in male and female cats were different, but statistical analysis showed no significant difference. This suggests that $C$. felis exposure in the cats in this study had no sex predilection, consistent with previous studies.

In addition to in cats, a high prevalence of Chlamydia has been found in growing pigs with or without clinical diseases [22], and chlamydial infections occur frequently in German sheep flocks, even in the absence of elevated abortion rates [23]. Reports of canine chlamydiosis are not common, possibly because $C$. felis is rarely considered to be a disease-causing pathogen in dogs. However, a few studies found chlamydial infections in clinically normal dogs [24,25]. For example, Pantchev et al. used species-specific real-time PCR assays and revealed that four of five dogs were infected by C. felis. The data of that study suggested that this species is highly adapted to cats and that despite the high prevalence of infection, C. felis-positive dogs, unlike cats, seldom suffer from conjunctivitis [25].

There is limited knowledge about chlamydiosis in dogs in China. Hence, we reported the prevalence of C. felis exposure in pet dogs in northwest China for the first time. In the present study, antibodies to C. felis were found in $32(12.1 \%)$ of 264 pet dogs, which is a higher prevalence than that detected in Dongguan (2.87\%), southern China [20]. Compared with other age groups, a higher prevalence of $C$. felis exposure was found in 2year-old cats; however, the difference was not statistically significant among different age groups $(P>0.05)$.

The results of this study revealed that $C$. felis is highly prevalent among the cats and pet dogs in Lanzhou, China, which suggests that this pathogen could be a significant cause of ocular diseases in these animals. Chlamydia felis is zoonotic pathogen, and maintenance of hygienic conditions and prompt treatment of affected cats and dogs is recommended to prevent human disease. The samples in the present study were collected between November 2010 and July 2011, which is a short period of time; thus, the obtained result may not reflect the actual situation of $C$. felis infection during longer periods. However, our results provide useful information for future studies. A number of factors may contribute to the varying seroprevalence, such as geographical conditions, diagnostic methods, feeding and living styles, and stress. In future studies, it would be interesting to test environmental samples such as soil, water, air, and 
ventilation systems to assess the contamination level of premises with C. felis.

\section{Conclusion}

The results of this investigation indicate the presence of C. felis infection in cats and pet dogs in northwestern China, which raises a public health concern because dogs and cats could be reservoir hosts of C. felis infection in humans. To our knowledge, this is the first report of $C$. felis infection in cats and dogs in northwest China.

\section{Competing interests}

The authors declare that they have no competing interests.

\section{Authors' contributions}

$\mathrm{XQZ}$ and SYH conceived and designed the study and wrote and critically revised the manuscript. SMW, SYH, and MJX performed the experiments, analyzed the data, and drafted the manuscript. DHZ and HQS helped in the study design, study implementation, and manuscript revision. All authors read and approved the final manuscript.

\section{Acknowledgements}

This work is supported in part by the Science Fund for Creative Research Groups of Gansu Province (Grant No. 1210RJIA006) and the Program for Outstanding Scientists in Agricultural Research to XQZ. We thank Edanz (www.edanzediting.com/bmc1) for improving the English language of this manuscript.

\section{Author details}

${ }^{1}$ State Key Laboratory of Veterinary Etiological Biology, Lanzhou Veterinary Research Institute, Chinese Academy of Agricultural Sciences, Lanzhou, Gansu Province 730046, PR China. ${ }^{2}$ College of Animal Science and Veterinary Medicine, Heilongjiang Bayi Agricultural University, Daqing, Heilongjiang Province 163319, PR China.

Received: 2 December 2012 Accepted: 17 May 2013

Published: 21 May 2013

\section{References}

1. Shewen PE: Chlamydial infection in animals: a review. Can Vet J 1980, 21(1):2-11.

2. Beeckman DS, Vanrompay DC: Zoonotic Chlamydophila psittaci infections from a clinical perspective. Clin Microbiol Infect 2009, 15(1):11-17.

3. Eidson M: Psittacosis/avian chlamydiosis. J Am Vet Med Assoc 2002, 221(12):1710-1712.

4. Entrican G, Wheelhouse NM: Immunity in the female sheep reproductive tract. Vet Res 2006, 37(3):295-309.

5. Pannekoek Y, Visser C, Duim B, Heddema ER: Chlamydophila psittaci infections in The Netherlands. Drugs Today (Barc) 2009, 45(Suppl B):151-157.

6. Verminnen K, Vanrompay D: Chlamydophila psittaci infections in turkeys: overview of economic and zoonotic importance and vaccine development. Drugs Today (Barc) 2009, 45(Suppl B):147-150.

7. Verweij PE, Meis JF, Eijk R, Melchers WJ, Galama JM: Severe human psittacosis requiring artificial ventilation: case report and review. Clin Infect Dis 1995, 20(2):440-442.

8. Travnicek M, Mardzinova S, Cislakova L, Valocky I, Weissova T: Chlamydial infection of cats and human health. Folia Microbiol (Praha) 2002, 47(4):441-444.

9. Longbottom D, Coulter LJ: Animal chlamydioses and zoonotic implications. J Comp Pathol 2003, 128(4):217-244.

10. Sykes JE: Feline chlamydiosis. Clin Tech Small Anim Pract 2005, 20(2):129-134

11. Arizmendi F, Grimes JE, Relford RL: Isolation of Chlamydia psittaci from pleural effusion in a dog. J Vet Diagn Invest 1992, 4(4):460-463.

12. Xu MJ, He Y, Liang R, Zhou DH, Lin RQ, Yin CC, He XH, Liang M, Zhu XQ: Seroprevalence of Chlamydia infection in pigs from intensive farms in southern China. J Anim Vet Adv 2010, 9(7):1143-1145.
13. Yang J, Ling Y, Yuan J, Pang W, He C: Isolation and characterization of peacock Chlamydophila psittaci infection in China. Avian Dis 2011, 55(1):76-81.

14. Huang SY, Wu SM, Xu MJ, Zhou DH, Danba C, Gong G, Zhu XQ: First record of Chlamydia abortus seroprevalence in Tibetan sheep in Tibet, China. Small Ruminant Res 2013. in press.

15. Zhang NZ, Zhou DH, Shi XC, Nisbet AJ, Huang SY, Ciren D, Wu SM, Zhu XQ: First report of Chlamydiaceae seroprevalence in Tibetan pigs in Tibet, China. Vector Borne Zoonotic Dis 2013. In press.

16. Zhou DH, Zhao FR, Xia HY, Xu MJ, Huang SY, Song HQ, Zhu XQ: Seroprevalence of chlamydial infection in dairy cattle in Guangzhou, southern China. Ir Vet J 2013, 66(1):2.

17. Lin RQ, Wang XQ, Yan C, He XH, Cheng T, Wang YN, Xu MJ, Yuan ZG, Zhang YB, Zhu XQ: Seroprevalence of Chlamydophila infection in chickens, ducks, geese and pigeons in Southern China. Afr J Microbiol Res 2011, 5(24):4240-4242.

18. Halánová M, Sulinova Z, Cislakova L, Trbolova A, Palenik L, Weissova T, Halan M, Kalinova Z, Holickova M: Chlamydophila felis in cats - are the stray cats dangerous source of infection? Zoonoses and Public Health 2011, 58(7):519-522.

19. Millán J, Rodriguez A: A serological survey of common feline pathogens in free-living European wildcats (Felis silvestris) in central Spain. Eur J Wildlife Res 2009, 55(3):285-291

20. Huang $B C H J, W u Z P, X i e ~ H Y, Z$, $h a n g X P$ : Investigation on serology of dogs and cats Chlamydia in Dongguan Guangdong. Chin J Vet Drug 2010, 44:9-10.

21. Stavisky J, Brennan M, Downes M, Dean R: Demographics and economic burden of un-owned cats and dogs in the UK: results of a 2010 census. BMC Vet Res 2012, 8(1):163.

22. Englund $\mathrm{S}$, af Segerstad CH, Arnlund F, Westergren E, Jacobson M: The occurrence of Chlamydia spp. in pigs with and without clinical disease. BMC Vet Res 2012, 8:9.

23. Lenzko H, Moog U, Henning K, Lederbach R, Diller R, Menge C, Sachse K, Sprague LD: High frequency of chlamydial co-infections in clinically healthy sheep flocks. BMC Vet Res 2011, 7:29.

24. Sprague LD, Schubert E, Hotzel H, Scharf S, Sachse K: The detection of Chlamydophila psittaci genotype C infection in dogs. Vet J 2009, 181(3):274-279.

25. Pantchev A, Sting R, Bauerfeind R, Tyczka J, Sachse K: Detection of all Chlamydophila and Chlamydia spp. of veterinary interest using speciesspecific real-time PCR assays. Comp Immunol Microbiol Infect Dis 2010, 33(6):473-484

\section{doi:10.1186/1746-6148-9-104}

Cite this article as: Wu et al: Chlamydia felis exposure in companion dogs and cats in Lanzhou, China: a public health concern. BMC Veterinary Research 2013 9:104.

\section{Submit your next manuscript to BioMed Central and take full advantage of:}

- Convenient online submission

- Thorough peer review

- No space constraints or color figure charges

- Immediate publication on acceptance

- Inclusion in PubMed, CAS, Scopus and Google Scholar

- Research which is freely available for redistribution 